\title{
MODELLING TORQUE GENERATION BY THE MERO-CARPOPODITE JOINT OF THE AMERICAN LOBSTER AND THE SNOW CRAB
}

\author{
S.C. MITCHELL and M.E. DEMONT* \\ Comparative Biomechanics Laboratory, Biology Department, \\ St. Francis Xavier University, Box 5000, Antigonish, Nova Scotia, \\ Canada, B2G 2 W5
}

(Received 8 July 2003; In final form 6 October 2004)

\begin{abstract}
The torque generated by a rotating joint comprises the useful force exerted by the joint on the external environment, and both the magnitude and distribution of torque through the step cycle during walking are important variables in understanding the mechanics of walking. The mechanics of the American lobster (Homarus americanus) and snow crab (Chionoecetes opilio) during walking were modelled to examine the relative roles of flexor versus extensor apodeme-muscle complexes, investigate which legs of these decapods likely contribute the greatest to locomotion, determine scaling effects of torque generation, and assess the relative roles of various model variables on torque production. Force generated along the length of the apodeme by the muscle was modelled based on apodeme surface area, muscle stress, and muscle fibre pinnation angle. Torque was then calculated from this estimated force and the corresponding moment arm. The flexor apodeme-muscle complex is calculated to generate consistently greater forces than the extensor, and generally this results in flexor torque being larger than extensor, though the snow crab does illustrate the opposite in two of its legs. This greater torque generation in flexion suggests that, in addition to the pushing of the trailing legs, the pulling action of the leading legs may play a significant role, at least during lateral walking. Leg 4 of both species appears to generate greater torques and thus provide the greatest forces for locomotion. Torque generation as a function of body size shows a second order response due to the increase in apodeme surface area. The pinnation angle of the muscle fibre is found to be insignificant in force generation, apodeme surface area (representing muscle cross sectional area) likely plays the most influential role in total force production, and moment arm controls the distribution of this force through the step cycle. Muscle stress remain a largely unknown quantity however, and may significantly affect both magnitude and distribution through step cycle of forces, and thus torque. Despite the uncertainty associated with the muscle stress parameter, the modelled results fit well with previously published force measurements.
\end{abstract}

Keywords: Model; Torque; Force; Locomotion; Lobster; Crab; Homarus; Chionoecetes

\section{INTRODUCTION}

Muscles generate the forces that rotate a joint which allow animal movement (Lieber, 1997). The contractile apparatus of the muscle fibre provides a linear force, and

*Corresponding author. Tel.: (902)867-5116. E-mail: edemont@stfx.ca 
this force is then translated to rotational force, or torque, via a fulcrum. This torque comprises the useful force generated by the joint (Goslow and van de Graf, 1982) and may be used by a walking animal to push off of, or pull along, the substrate in order to translate the body. Lobsters and crabs move easily either forward/backward or laterally (Clarac 1981, 1984; Evoy and Ayers, 1982; Chasserat and Clarac, 1983). During forward walking by the lobster, the mero-carpopodite joint remains at a fixed angle (Macmillan, 1975; Evoy and Ayers, 1982; Mitchell and DeMont, 2003b), while during lateral walking by crustaceans this joint undergoes large angles of excursion (Ayers and Davis, 1977; Sleinis and Silvey, 1980; Jamon and Clarac, 1997). This movement of the limb about the joint generates different forces on the two sides of the laterally walking animal, with the trailing side pushing and the leading side decelerating the body (Blickhan and Full, 1987) or generating a pulling force (Sleinis and Silvey, 1980). The fixed angle of the mero-carpopodite joint maintained by the lobster during forward walking may also be explainable in terms of torque, if it can be shown that the observed joint angle maximizes torque generation. Therefore, both the magnitude and distribution of torque throughout the step cycle are likely significant variables in understanding the mechanics of crustacean walking.

Lobsters and crabs have pinnated muscle architecture in their walking legs with the extension of the leg being powered by a single extensor muscle and the flexor by two muscles - a main and a very small accessory flexor (Macmillan and Dando, 1972; Macmillan, 1975; Ayers and Davis, 1977; Ayers and Clarac, 1978). According to a model presented by Alexander $(1969,1983)$ the force generated by a pinnate muscle system may be estimated from knowledge of the physiological cross-section of the muscle, the pinnation angle of the muscle fibres and the stress (force per unit area) exerted by the muscle. The physiological cross section of the muscle may be estimated from the surface area of the apodeme and the pinnation angle measured directly. Assigning a reasonable muscle stress value is more problematic, yet selection of this will significantly affect resulting force estimates. From the modelled force it is then straightforward to calculate torque as the product of this force and the moment arm (the perpendicular distance from the line-of-action to the fulcrum) of the apodeme. An understanding of the walking kinematics of the animal then allows calculation of forces and joint torques throughout the step cycle.

The muscle architecture, morphometrics, kinematics and allometry of the merocarpopodite joint have been described previously (Mitchell and DeMont, 2003a, b). From this work it is possible to model the forces generated by the extensor and flexor muscles, and the torque produced as the joint moves through its excursion. By modelling the forces and torques generated during walking by the lobster and snow crab, it is possible to determine: (i) which component of the mero-carpopodite joint, the flexor apodeme-muscle complex or the extensor, generates the greater torque, and hence contributes most to locomotion, (ii) whether there exist anatomical specialization of structures within the various walking legs along the length of the animal's body which contribute to specialization of leg function, and (iii) if torque generation scales proportionately with size of the animal. In addition, the modelling procedure allows us to assess the relative contribution of the different model variables to force and torque generation, thereby determining which are most significant. 


\section{MATERIALS AND METHODS}

Torque generation about the mero-carpopodite (M-C) joint of the American lobster (Homarus americanus, H. Milne Edwards) and snow crab (Chionoecetes opilio, O. Fabricius) was modelled during joint excursion from joint angles of $\sim 25^{\circ}$ to $165^{\circ}$ as (adapted from Alexander, 1969, 1983):

$$
\hat{\tau}_{k}=m a_{k} \cdot \sum_{i=1}^{8} 2 \cdot S A_{\text {apod }, i} \cdot \sigma \cdot \cos \theta_{i}
$$

where $\hat{\tau}_{k}$ is the estimated torque $(\mathrm{Nmm})$ and $m a_{k}$ is the moment arm $(\mathrm{mm})$ at joint angle $k, S A_{\text {apod, } i}$ is the apodeme surface area $\left(\mathrm{mm}^{2}\right)$ for increment $i$ (see below), $\theta_{i}$ represents the pinnation angle of muscle fibres attaching to the apodeme at increment $i$, and $\sigma$ is the muscle stress during contraction. The summed terms in Eq. (1) represent the total force applied along the line-of-action (i.e., the apodeme) by the muscle fibres and this represents the force acting at the point of attachment of the apodeme to the carpopodite (see Fig. 1 in Mitchell and DeMont, 2003a).

The moment arm, apodeme surface area, and muscle fibre pinnation angle required for Eq. (1) were measured and reported previously (see Mitchell and DeMont, 2003a,b). A muscle stress value of $10 \mathrm{kNm}^{-2}$ was selected for use based upon values reported in the literature. Representative values of crustacean muscle stresses are presented in Table I, and it is apparent that different muscles within a single organism can have order-of-magnitude differences in stress values. This variability, combined with a lack of knowledge of the magnitude of the stress developed by a muscle during regular activity, i.e., non-maximal contraction, makes any selection of this value for the model somewhat arbitrary (see also Discussion).

The apodeme surface area, representing muscle cross sectional area, was estimated for various sized lobsters and snow crabs based on previously reported regression equations of apodeme area, as a function of carapace length (lobster) or width (crab). The apodeme was divided into eight increments $(i)$, each representing $12.5 \%$ of the total apodeme length. Due to muscle fibres not covering the entire apodeme surface (e.g., Bennet-Clark, 1975) or part of the area being attached by non-contractile

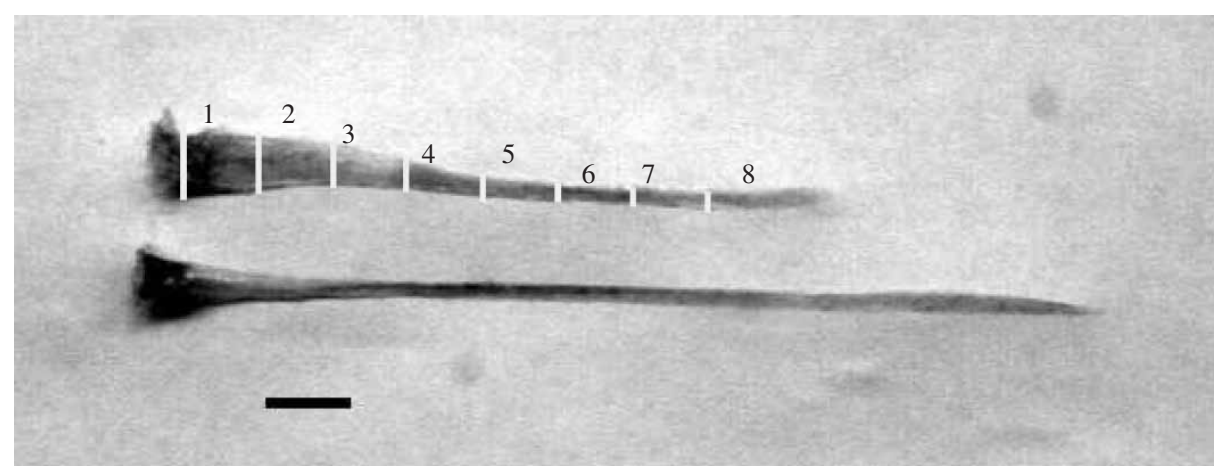

FIGURE 1 Division of apodeme into eight increments $(i=1-8)$ for purposes of modelling muscle cross sectional area. Upper apodeme is extensor; lower apodeme is flexor. Scale bar $=1 \mathrm{~cm}$. 
TABLE I Sample of muscle fibre stress values $\left(\mathrm{kNm}^{-2}\right)$ reported in the literature for various crustacean muscles

\begin{tabular}{|c|c|c|c|c|}
\hline \multicolumn{5}{|c|}{ Muscle fibre type } \\
\hline Structure & Slow & Fast & Unspecified & Source \\
\hline \multicolumn{5}{|l|}{ Leg } \\
\hline Homarus americanus & 5.52 & 6.9 & & Jahromi and Atwood (1971) \\
\hline H. americanus & $5.8-6.05$ & & & Sherman and Atwood (1971) \\
\hline Astacus fluviatilis & & $548-1131$ & & Zachar and Zacharova (1966) \\
\hline \multicolumn{5}{|l|}{ Abdomen } \\
\hline H. americanus & $118-442$ & $21-83$ & & Jahromi and Atwood (1969) \\
\hline Orconectes virilis & & & $>14$ & Webb (1979) \\
\hline \multicolumn{5}{|l|}{ Scaphognathite } \\
\hline Carcinus maenas & & & $13-122$ & $\begin{array}{l}\text { Josephson and Stokes (1987, } \\
1989,1999)\end{array}$ \\
\hline \multicolumn{5}{|l|}{ Chelae } \\
\hline H. americanus & & & 430 & Elner and Campbell (1981) \\
\hline Callinectes sapidus & & & $450-600$ & Govind and Blundon (1985) \\
\hline Cancer pagurus & & & 721 & Warner and Jones (1976) \\
\hline Cancer species ${ }^{\mathrm{a}}$ & & & $383-1535$ & Taylor (2000) \\
\hline Menippe mercenaria & & & $673-2200$ & Blundon (1988) \\
\hline C. maenas & & & 1057 & Warner et al. (1982) \\
\hline
\end{tabular}

${ }^{a}$ Cancer antennarius, C. branneri, C. gracilis, C. magister, C. oregenensis, C. productus.

components such as fat, connective-tissue or mitochondria (Richmond, 1998), the first increment of the apodeme was considered to have less muscle connection than the remainder. Calculated total apodeme area from the regression was multiplied by factors of 0.06 in this first increment and 0.125 for each of the remaining seven increments. This resulted in a distribution of apodeme area as half the available area in the first increment, and increments 2-8 treated as rectangles with full muscle coverage (see Fig. 1). This distribution amounts to the modelled apodeme (i.e., that with muscle attachment) as $93.5 \%$ of the total apodeme surface area. The calculated surface area was then multiplied by two to account for muscle attachment to both dorsal and ventral surfaces. The extensor and main flexor apodeme muscle complexes were used in these analyses; the accessory flexor is a small muscle and is not likely to contribute significantly, compared to the much larger main flexor. The accessory flexor was therefore, omitted. Muscle fibre pinnation angle varies along the length of the apodeme (Mitchell and DeMont, 2003a) and this was modelled by linear interpolation of the pinnation angle at each increment $i$ along the apodeme.

Moment arm estimates used in Eq. (1) are mean moment arms at $15^{\circ}$ increments through the joint angle of excursion for each of a range of lobster and crab sizes (see Mitchell and DeMont, 2003b for details). Due to the lack of rigorous statistical functions of moment arm as a function of size (i.e., $r^{2}$ of moment arm regressed on body size $0.29-0.51$ for lobster, $0.35-0.85$ for snow crab; see Mitchell and DeMont, 2003b) suggesting that there is not a strong relationship between moment arm and body size, the mean moment arm across the range of body sizes was used in place of a size dependant value. Multiplication of these mean moment arms by the calculated force along the line-of-action at each of the $15^{\circ}$ increments through the joint excursion allow estimation of the distribution of torque during $\mathrm{M}-\mathrm{C}$ joint flexion/extension in these animals. 
With the exception of $\sigma$, the variables of the model have been measured and these measurements involve inherent variance as well as measurement error. The propagation of these errors through successive calculations to provide final error estimates on calculated torques was done using the equation (modified from Bevington, 1969):

$$
\hat{\varepsilon}_{k}=\sqrt{\hat{\tau}_{k} *\left[\operatorname{Var}\left(m a_{k}\right) / m a_{k}^{2}+\operatorname{Var}\left(\theta_{i}\right) / \theta_{i}^{2}+\operatorname{Var}\left(S A_{\mathrm{apod}, i}\right) / S A_{\mathrm{apod}, i}^{2}\right]}
$$

where $\hat{\varepsilon}_{k}$ is the error associated with the estimate of $\hat{\tau}_{k}$ for each $k$ joint angle and $\operatorname{Var}()$ is the variance associated with the variable in brackets.

In order to assess the accuracy/validity of these modelling efforts, results for the lobster were compared with those in published studies. Due to crustacean size in the literature being largely reported as body mass (g), but the developed models using carapace size as the body size variable, a power regression was conducted on the animals used in this study to relate carapace size to body mass. This regression resulted in:

$$
\begin{aligned}
& \text { Lobster carapace length }(\mathrm{mm})=11.785 \operatorname{mass}^{0.3196}(\mathrm{~g}) \\
& r^{2}=0.9799, \quad n=38, \quad \text { range }=299-12.868 \mathrm{~g}
\end{aligned}
$$

To estimate the force of the dactyl on the environment $\left(F_{2}\right)$ as part of the validation, the calculated joint torque $\left(\hat{\tau}=F_{1} * L_{1}\right)$ was used as:

$$
F_{2}=\left(F_{1} * L_{1}\right) / L_{2}
$$

where $L_{2}$ is the length from the M-C joint to the dactylopodite. $L_{2}$ was estimated as a function of carapace size using regression analysis. The force $\left(F_{2}\right)$ exerted by the joint was then added to the downward force of the animals mass (estimated as $10 \%$ of the out-of-water mass; see Clarac and Chasserat, 1983; Martinez et al., 1998) divided by the number of legs supporting that mass (assumed to be seven, one leg stepping) to derive a total downward directed force of the body mass plus that generated by the leg muscles. These estimated forces could then be compared directly with the few published direct measurements.

\section{RESULTS}

Prior to examining torque production as a function of body size, forces and torques generated by the legs of representative sized lobster and snow crab are presented. The mean carapace length for the lobster $(99 \mathrm{~mm})$ and carapace width for the snow crab $(85 \mathrm{~mm})$ of all animals investigated (see Mitchell and DeMont, 2003a,b for details on animals) are used to illustrate the following results.

The estimated force generated for a given muscle stress, as summed along the length of the apodeme to the carpopodite junction, remains approximately constant throughout the movement of the $\mathrm{M}-\mathrm{C}$ joint through its angle of excursion (Fig. 2). The difference in force generation due to changing pinnation angle generally amounts to less than $5 \%$. This implies that the change in pinnation angle, the only variable changing, is insignificant with respect to force generation 
(a)

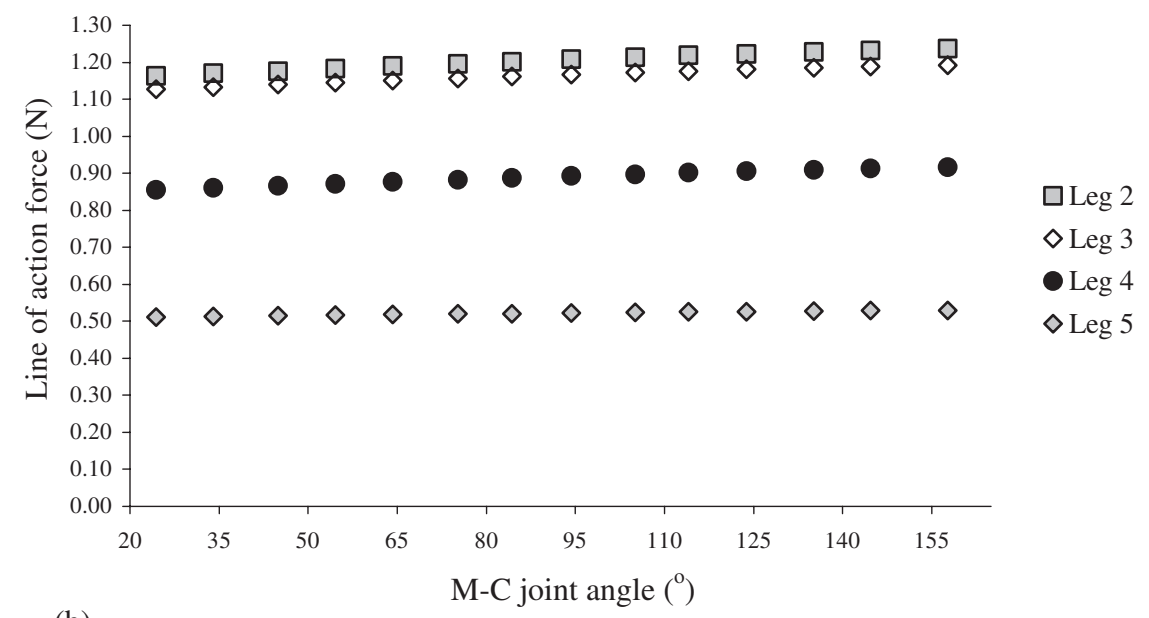

(b)

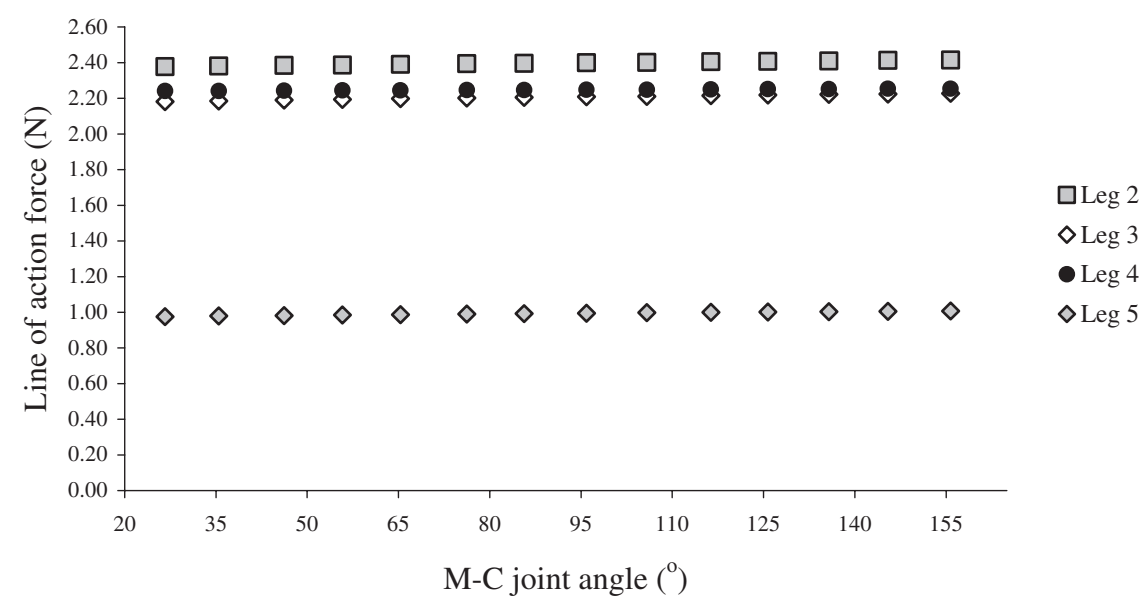

FIGURE 2 Calculated forces of flexor apodeme-muscle complex for the four walking legs of (a) the lobster (carapace length $=99 \mathrm{~mm}$ ); (b) the snow crab (carapace width $=85 \mathrm{~mm}$ ).

in these crustaceans during joint movement. The estimated forces generated by the extensor apodeme-muscle complex of legs $2-5$ of the lobster are very similar (i.e., $0.7-1.0 \mathrm{~N}$ at $\sigma=10 \mathrm{kNm}^{-2}$ ), with leg 4 generating the higher value forces and leg 5 the lower values. The flexor apodeme-muscle complex of these animals show legs 2 and 3 generating greater forces for a constant stress than leg 4, which in turn is greater than leg 5 (Fig. 2a). Legs 2 and 3 appear to generate greater forces in flexion than extension, while the posterior two leg pairs generate either similar (leg 4) or lesser forces (leg 5). Within the legs of the snow crab the extensor apodeme-muscle complex of legs 2 and 3 generate similar estimated forces $(\sim 1.8 \mathrm{~N})$ and these are greater than leg $4(\sim 1.4 \mathrm{~N})$, which are considerably greater than leg $5(\sim 0.8 \mathrm{~N})$. The flexor apodeme-muscle complex shows legs $2-4$ generating similar forces, and these are all over double that generated by leg 5 (Fig. 2b). 
Leg 2

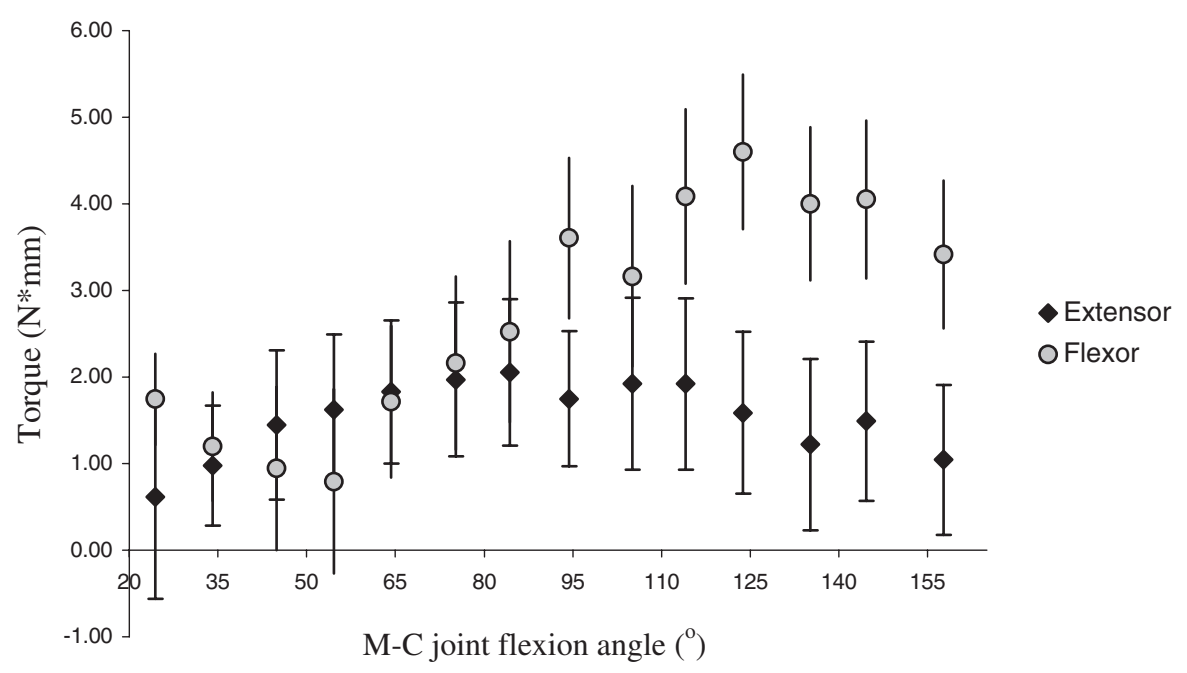

Leg 3

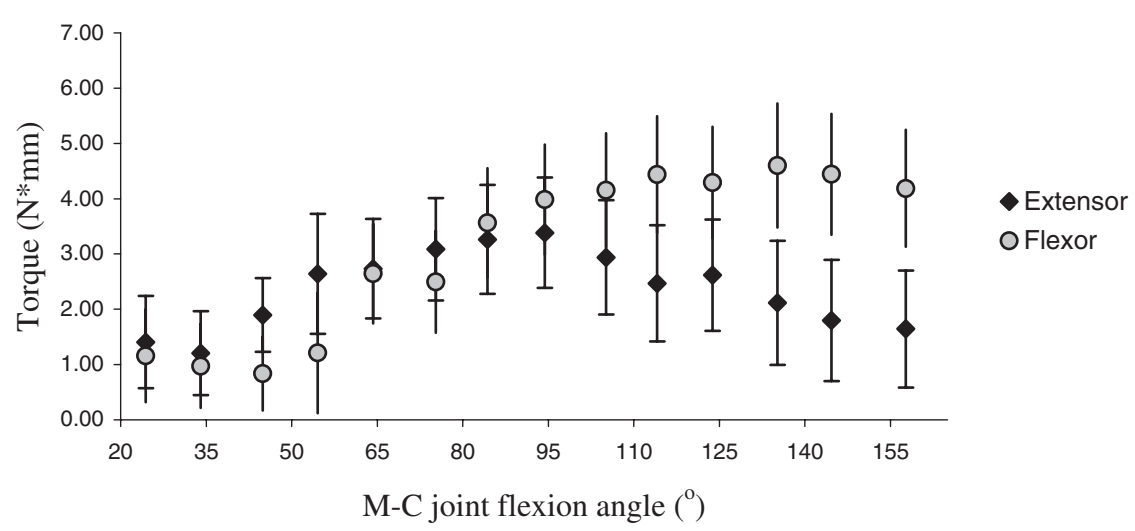

FIGURE 3 Calculated torque generated by legs $2-5$ of the lobster (carapace length $=99 \mathrm{~mm}$ ). Error bars are propagated error (see Eq. (2)).

The flexor apodeme-muscle complex in the crab is structurally able to consistently generate greater forces than the extensor apodeme-muscle complex, for a given muscle stress.

The distribution of torque generated as the joint moves through its angle of excursion closely follows the moment arm (see Mitchell and DeMont, 2003b) and the modelled flexor apodeme-muscle complex generally produces greater torque than the extensor. However, in the lobster there is an increase in relative torque by the extensor apodeme-muscle complex compared with the flexor moving from legs 2 to 5 (Fig. 3). This may be due to the distribution of the flexor torque through joint flexion remaining constant between the four legs, while the extensor shows some variability, increasing in magnitude at lower flexion angles in legs 4 and 5. The greatest magnitude of 
Leg 4

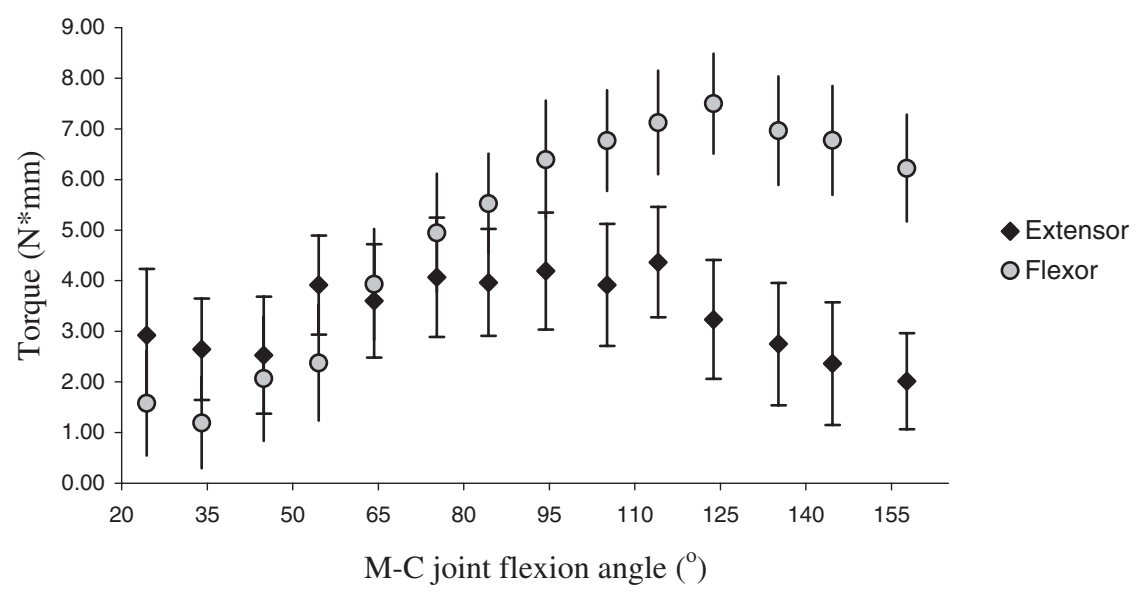

Leg 5

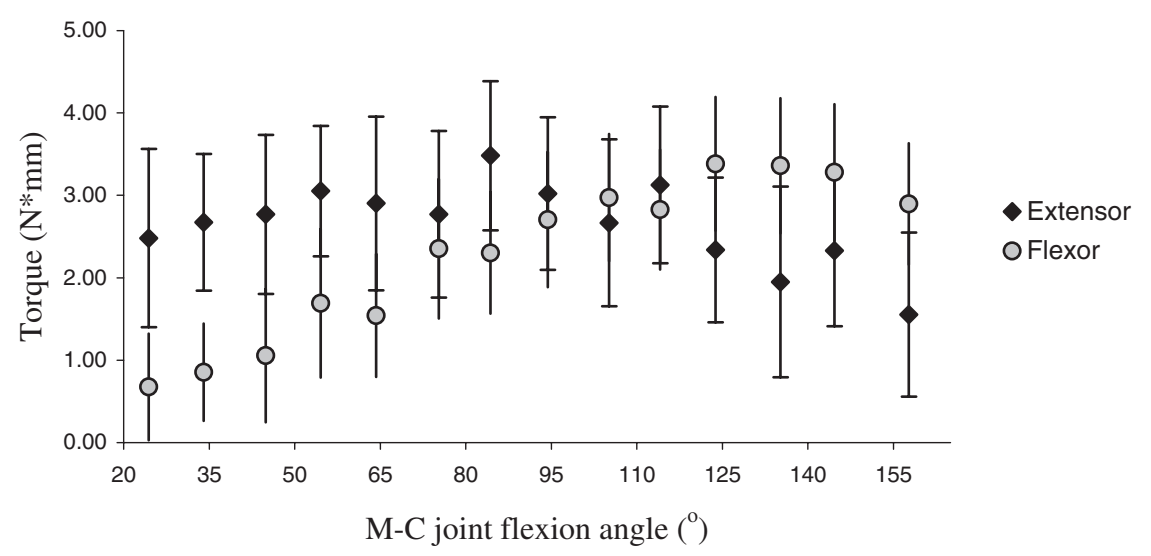

FIGURE 3 Continued.

torque in the lobster are associated with leg 4 (flexor maximum $\sim 7 \mathrm{Nmm}$, extensor maximum $\sim 4 \mathrm{~N} \mathrm{~mm}$ ). In the snow crab the extensor apodeme-muscle complex appears to generate much greater torques relative to the flexor than in the lobster; indeed, legs 3 and 5 show the extensor developing greater torques than the flexor (Fig. 4). Similar to the lobster, the flexor pattern of torque appears relatively constant, while it is the extensor apodeme-muscle complex which exhibits the variability, accounting for the changing relative importance. In this crustacean, the greatest magnitude of torque is associated with legs 2 and 4 (flexor maximum $\sim 12-14 \mathrm{~N} \mathrm{~mm}$ ) and legs 4 and 5 (extensor maximum $\sim 8-10 \mathrm{Nmm}$ ). Figure 5 illustrates calculated torque for leg 3 of a laterally walking crab with observed joint kinematics. The lower panel in this figure presents the stepping cycle of the crab (from Mitchell and DeMont, 2003b) and the upper panel shows calculated torque generated for each of the extensor and flexor 
Leg 2

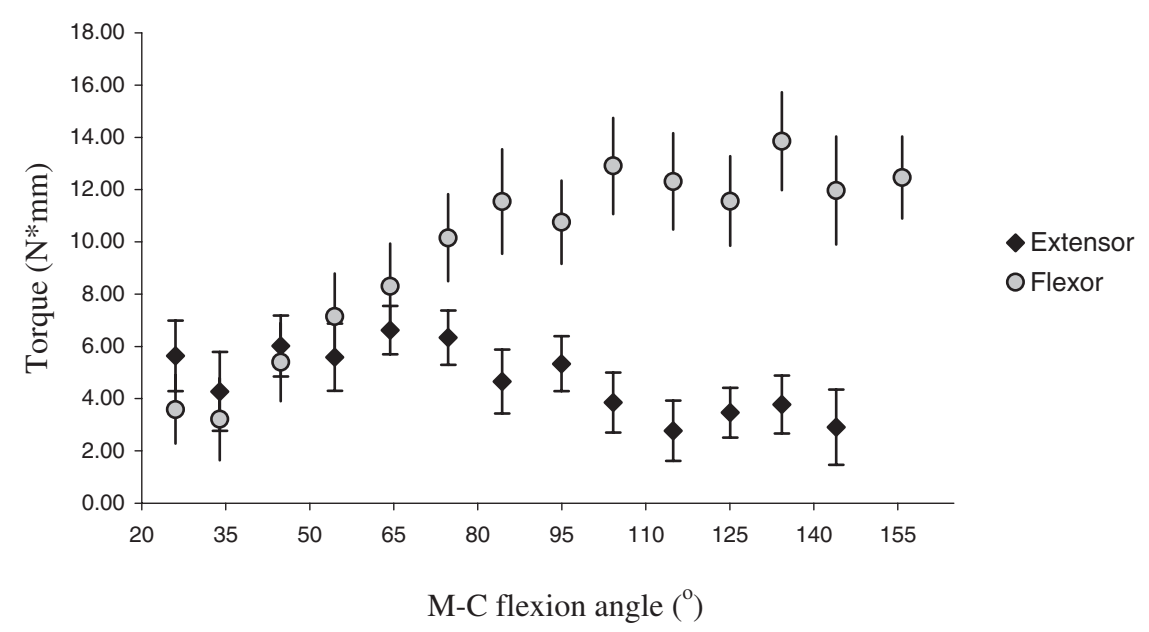

Leg 3

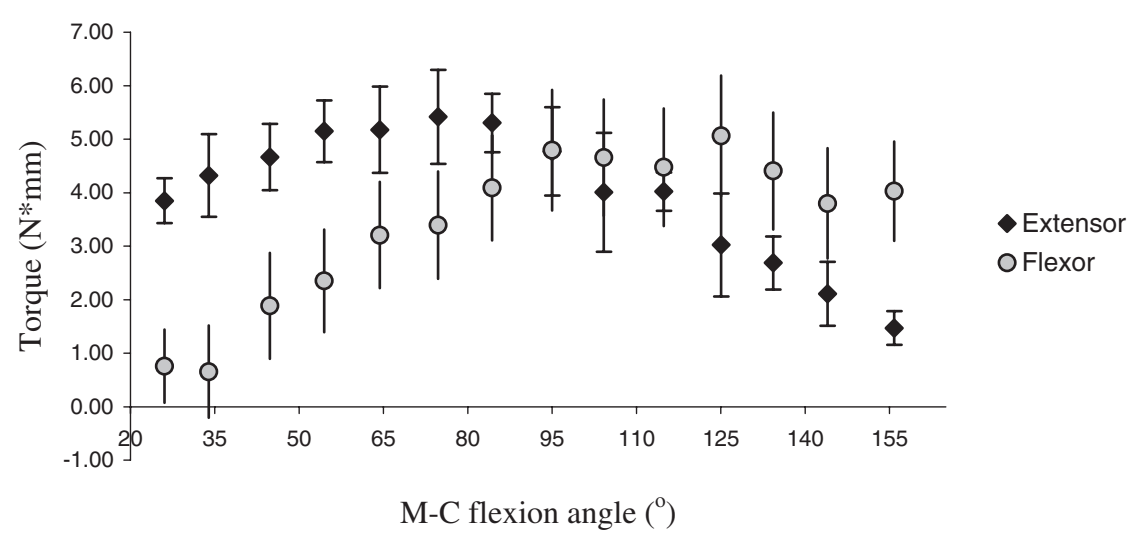

FIGURE 4 Calculated torque generated by legs $2-5$ of the snow crab (carapace width $=85 \mathrm{~mm}$ ). Error bars are propagated error (see Eq. (2)).

using the model here. As the muscle activation and behavior is unknown during relaxation, these areas are left as gaps in the figure. From this figure it may be seen that the flexor torque generally rises rapidly, then declines as the joint is flexed (i.e., as the joint angle goes from $\sim 140^{\circ}$ to $80^{\circ}$ ), while the extensor exhibits a more linear decline from a maximum at small joint flexion angles $\left(\sim 80^{\circ}\right)$ to a minimum at full extension $\left(\sim 140^{\circ}\right)$.

Calculated maximum torque as a function of carapace size illustrates a power curve response with body size (Table II). This maximum torque is the maximum value taken by the apodeme-muscle complex, at a constant muscle stress value, through the joint angle of excursion, and was calculated for each of the $5 \mathrm{~mm}$ increments in carapace size over the range of sizes of animals used in this study (see Mitchell and DeMont 2003a,b for details). The crabs illustrate a greater curvature to their response (i.e., exponent between 2.335 and 2.769) compared with the lobster (exponent between 1.787 
Leg 4

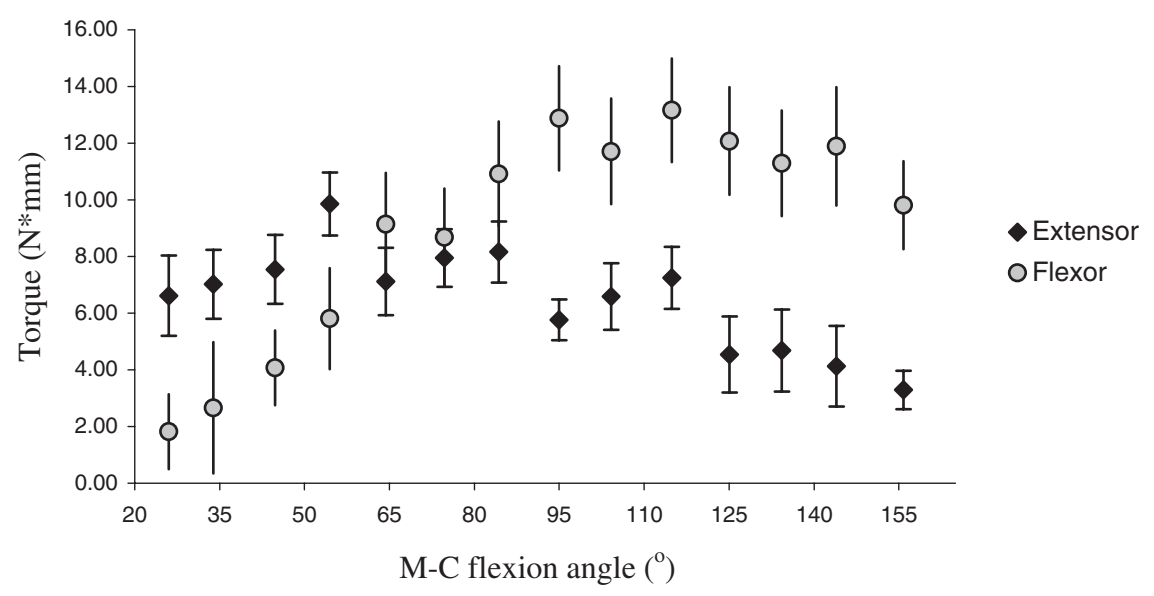

Leg 5

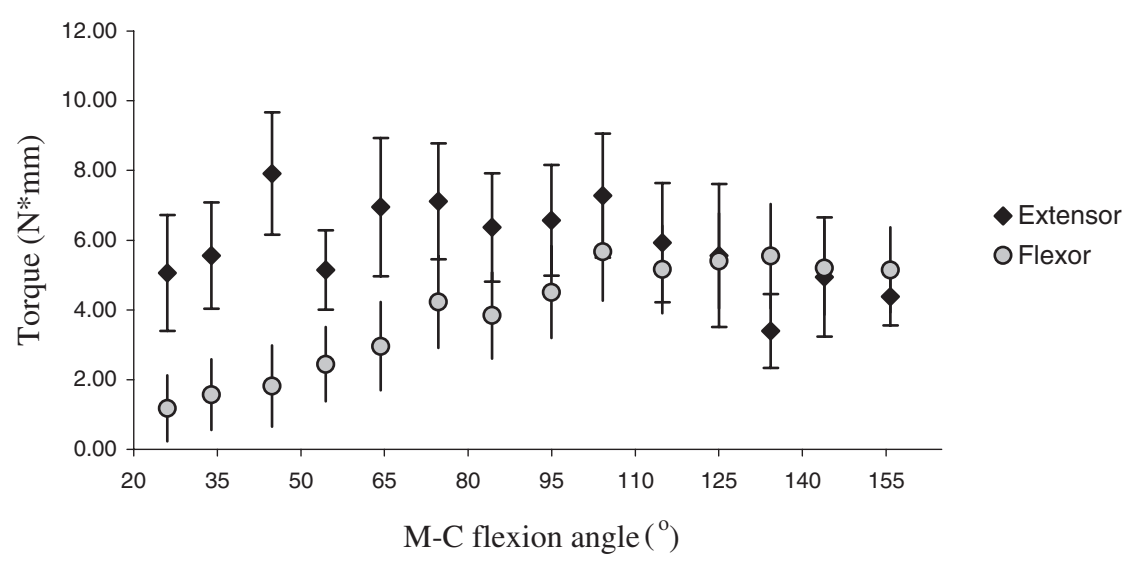

FIGURE 4 Continued.

and 2.42). This suggests that all legs for both species show scaling with negative allometry of maximum torque development with body size (isometric values given in Table II). The isometric value is estimated by using the established relation that force increases proportionally with muscle cross sectional area (Gans, 1982; Richmond, 1998, and others) and this variable (e.g., apodeme surface area) has been shown to be a function of carapace size in these two crustaceans (Mitchell and DeMont, 2003a). The scaling exponent from that relationship is then increased by a value of 1.0, as this is the expected isometric scaling value for the moment arm (force ${ }^{\alpha} *$ moment $\operatorname{arm}^{\beta}=$ torque $^{\alpha+\beta}$, where $\alpha$ is the scaling exponent of apodeme surface area with carapace size and $\beta$ is 1.0) in order to estimate the isometric condition of torque. From this it appears that larger animals generate disproportionately lesser torque given the same muscle stress than smaller animals; though it is, of course, much greater in magnitude.

The calculated torques were decomposed into forces applied to the external environment (Eqs. (3) and (4)) in order to allow comparison with the limited published data. 

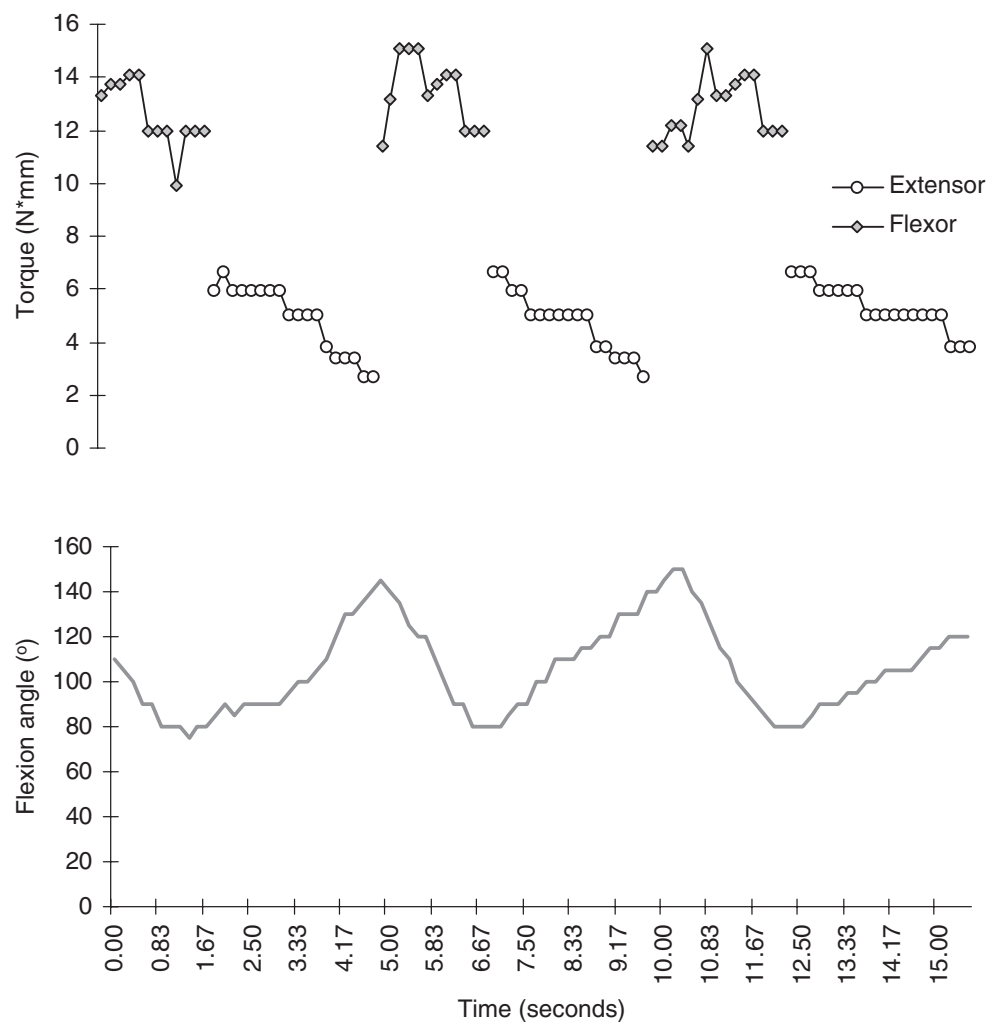

FIGURE 5 Time course of mero-carpopodite joint angle through stepping cycle of leg 3 of the snow crab (lower trace) and simultaneous calculated torque for extensor and flexor apodeme-muscle complexes (upper trace). Model crab was $85 \mathrm{~mm}$ carapace width with mean muscle stresses $( \pm \mathrm{SD})$ of: extensor $=1.962 \mathrm{~N}$ $(0.0144)$, flexor $=2.206 \mathrm{~N}(0.015)$. See text for details.

TABLE II Calculated power relationships between maximum torque $(y=\mathrm{Nmm})$ and carapace size $(x=\mathrm{mm})$ for each apodeme-muscle complex of each leg for the lobster and snow crab. See text for derivation of isometric exponent

\begin{tabular}{llll}
\hline Leg & Apodeme & \multicolumn{1}{c}{ Equation } & Isometric exponent \\
\hline Lobster & & & 3.7 \\
2 & Extensor & $y=3.044 * 10^{-5} x^{2.420}$ & 3.2 \\
& Flexor & $y=1.300 * 10^{-4} x^{2.279}$ & 3.5 \\
3 & Extensor & $y=9.186 * 10^{-4} x^{1.787}$ & 3.4 \\
& Flexor & $y=2.400 * 10^{-4} x^{2.146}$ & 3.1 \\
4 & Extensor & $y=3.828 * 10^{-4} x^{2.033}$ & 3.2 \\
5 & Flexor & $y=2.867 * 10^{-4} x^{2.213}$ & 3.3 \\
& Extensor & $y=6.852 * 10^{-4} x^{1.857}$ & 3.4 \\
Snow Crab & Flexor & $y=2.3368 * 10^{-4} x^{2.085}$ & 3.9 \\
2 & Extensor & & 4.0 \\
3 & Flexor & $y=4.818 * 10^{-5} x^{2.663}$ & 3.8 \\
& Extensor & $y=1.253 * 10^{-4} x^{2.614}$ & 3.6 \\
4 & Flexor & $y=4.405 * 10^{-5} x^{2.638}$ & 3.9 \\
5 & Extensor & $y=3.556 * 10^{-5} x^{2.671}$ & 3.9 \\
& Flexor & $y=4.483 * 10^{-5} x^{2.769}$ & 3.2 \\
& Extensor & $y=4.112 * 10^{-4} x^{2.335}$ & 3.2 \\
\hline
\end{tabular}




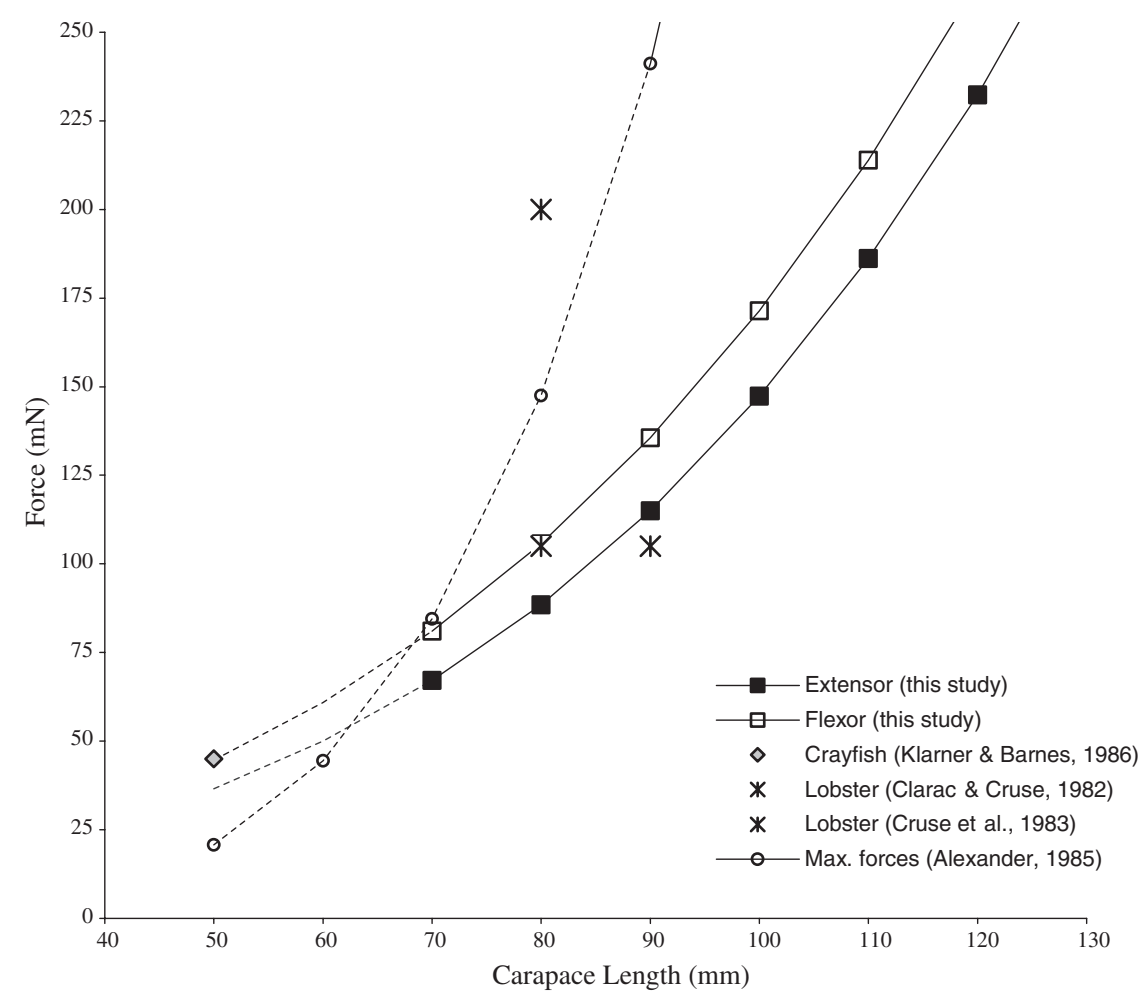

FIGURE 6 Force on environment as a function of carapace size for leg 4 of the lobster. The line from

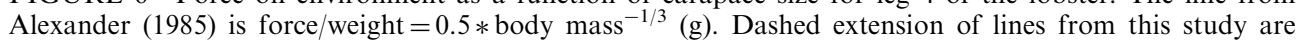
extrapolation outside range of measured values.

These are presented in Fig. 6 and indicate a good fit between modelled results and other reported values. The fit between the crayfish of Klärner and Barnes (1986) and the lobster of Clarac and Cruse (1982) is particularly encouraging for lending support to the modelled results.

\section{DISCUSSION}

The validation of the modelling results with published measured values, and Alexander's (1985) estimates of maximum force exerted on the environment, suggests that these results accurately estimate forces exerted on the substrate and, by extension, the torques generated about the $\mathrm{M}-\mathrm{C}$ joint. This provides a level of confidence in basing conclusions on these results, despite significant uncertainties in the muscle stress parameter of the model (see below).

The force generated at the apodeme-carpopodite junction appears to be very stable through joint extension/flexion, and variable between legs when using a constant muscle stress. The change in pinnation angle that these muscles undergo during contraction appears to be insignificant in terms of force production; this is in keeping with previous findings (Gans, 1982; Richmond, 1988; Ledoux et al., 2001). The analysis 
of pinnation angles reported here has functionally confirmed what was deduced based on structural information. The patterns of force production between apodemes and legs in both the lobster and snow crab - i.e., flexor apodeme-muscle complexes generally generating greater forces, inter-leg differences for the same apodeme-muscle complex - are consistent with patterns of apodeme surface area described by Mitchell and DeMont (2003a), confirming that the apodeme surface area plays a highly influential role in force development. The influence of the remaining variable, muscle stress, on force production is much more difficult to assess.

A muscle stress value of $10 \mathrm{kN} \mathrm{m}^{-2}$ was selected for the model. It may be seen from Table I that this is, in fact, at the lower end of stress characteristics for crustacean muscle fibres. A low value was selected as it may be expected from energetic considerations that during normal walking an animal would not contract the muscle to maximal tension. The walking leg muscles of the lobster and other crustaceans are made up of muscle fibres with individual contraction characteristics (i.e., fast, slow, and intermediate fibres; Jahromi and Atwood, 1971; Atwood, 1973; Govind, 1995). In addition to individual fibre characteristics, the relatively complex neuromuscular system of crustaceans - involving multi-terminal innervation of single fibres, electrical continuity between fibres, and neuron-neuron inhibition - governs the strength of the stimulation received by the fibre (Prosser and Brown, 1962; Atwood, 1972, 1973; Govind, 1995). Atwood (1972), Gans (1982), and Gans and DeVree (1987) provide lists of factors affecting force production. The most relevant here are sarcomere length, mitochondrial content, physiological cross section, position of sarcomeres on the length-tension curve, the time course of force application, and the frequency at which the fibre is stimulated. Clearly, selecting a single stress value for such a complex and time-varying system greatly oversimplifies the true nature of the muscle. Values of $\sigma$ used for modelling of crustacean muscle force during locomotion have ranged from $10 \mathrm{kN} \mathrm{m}^{-2}$ (Daniel and Meyerhöfer, 1989) to $200 \mathrm{kN} \mathrm{m}^{-2}$ (Blickhan et al., 1993) reflecting this uncertainty.

A further important simplification of the model is the omission of a time course of muscle contraction. The muscle stress is represented as a constant value without an initial build up of tension during the beginning of contraction nor relaxation at the end. Due to the previously mentioned complexity of muscle fibre stimulation, and a lack of documentation on the rate of recruitment of muscle in the contracting apodeme-muscle complex during regular walking cycles in crustaceans, the development of contractile tension through the step cycle is ignored here. It may be expected that the time required to build to contraction and relax from contraction (i.e., its length tension curve), would decrease the calculated torques at the extreme ends of the joint excursion, but the central region - where the muscle has likely reached maximum tension development for that stimulation magnitude/frequency - will be less influenced and may exhibit a relatively constant contraction through that period. The selection of an appropriate $\sigma$ however, remains contentious as values over an order-of-magnitude may be justified, but will result in very different estimates of force for a given system.

The distribution of torques through $\mathrm{M}-\mathrm{C}$ joint excursion follows the pattern of moment arm development described for these crustaceans (Mitchell and DeMont, 2003b). Given that the other influential model variables were treated as constants, this is not surprising. The flexor apodeme--muscle complex generally generates greater torque than the extensor, though in the snow crab the extensors of legs 3 and 5 generate greater torques than the flexor apodeme-muscle complex. This, despite the 
force along the apodeme line-of-action being less for the extensor than for the flexor. This illustrates the importance of the moment arm as a structure that can compensate for decreased force and still provide equal or greater torque. The change in relative torque production by the extensor and flexor apodeme-muscle complexes in the lobster between legs 2 to 5 implies the first two legs are adapted to maximize flexion (i.e., pulling) forces, leg 4 equalizes extension (i.e., pushing) and flexion, and leg 5 produces greater extension torques than flexion. Leg 4 of the lobster generates the greatest magnitude of torque among the legs; this is consistent with the center of mass being located close to these legs (Macmillan, 1975; Nauen and Shadwick, 1999) and thus they provide much of the support of the animal in addition to locomotion. The forward walking lobster maintains its M-C joint at specific angles - leg $3 \sim 58^{\circ}$, leg $4 \sim 91^{\circ}$, and leg $5 \sim 55^{\circ}$ (Mitchell and DeMont, 2003b). At these angles, the torque generated by the extensor apodeme-muscle complex of legs 3 and 5 exceeds that generated by the flexor; leg 4 shows the flexor producing greater torque than the extensor. This may be expected to increase the stability of the animal as the three legs over which the center of mass is placed form a tripod with legs 3 and 5 pushing out, and leg 4 pulling in.

The calculated torque plotted together with the step cycle in Fig. 5 further illustrates the importance of the moment arm, as this provides an initial high torque value either at the beginning of flexion or extension and a subsequent decline. The much more regular, linear decline in torque by the extensor versus the flexor is probably due to the less peaked or curved moment arm exhibited through joint flexion relative to the flexor (see Mitchell and DeMont, 2003b). The overall image from this figure however, is that the flexor apodeme-muscle complex appears to generate greater forces, but flexion occurs over a shorter portion of the step cycle than the lower torque generating extension. This is consistent with suggestions that crabs use their leading legs to examine the substrate for traction and foot placement (Hui, 1992).

The extensor apodeme-muscle complex of the snow crab shows greater torque than the flexor within legs 3 and 5. However, the absolute magnitude of these torques are less than legs 2 and 4. Leg 5 does not likely contribute to a large extent in lateral walking in this species as it is considerably smaller than the other three walking legs (see discussion, Mitchell and DeMont, 2003b). It appears that legs 2 and 4 are the primary force producing legs, and they do so maximally in flexion. Therefore, as suggested earlier (Mitchell and DeMont, 2003b) the snow crab may utilize the flexion action to a greater degree than extension in lateral walking. For each species, it appears that leg 4 generates the greatest torque suggesting that this leg may provide a large portion of the propulsive power during walking in these crustaceans. This finding is in keeping with that reported for other lobster (Clarac and Chasserat, 1983) and crayfish (Pond, 1975; Klärner and Barnes, 1986; Jamon and Clarac, 1997).

The approximately second order curves generated when maximum torque is treated as a function of carapace size illustrate a highly non-linear response of torque with animal size. This may be interpreted as due solely to the apodeme surface areas, as pinnation angle has been shown to be insignificant, the muscle stress is common for all sizes, and the same mean moment arms were used for each size. Therefore, only the apodeme surface area varies in this analysis. The good fit of the calculated line (Fig. 6) to the crayfish and lobster, suggests that the modelling is reasonable and thus variations in torque may be largely ascribed to apodeme surface area. However, it should be noted that the use of a mean moment arm, 
rather than a size-dependent function, may underestimate torques for small animals and overestimate for the large.

All of the variables of Eq. (1) may not be expected to be equally significant in force and torque production. Pinnation angle has already been shown to be of little influence on force generation. The role of the muscle cross section (apodeme surface area) is highly influential as may be noted from the functions of torque versus body size in Table II. At constant muscle stress, and using the same mean moment arm across sizes, torque shows a second order response with size. That is, it scales approximately with the dimension of apodeme area. Therefore, changes in apodeme surface area may be a very efficient way for the animal to increase force and torque production. Changes in muscle stress will alter the resulting output force in a linear manner and so the increase will not be as dramatic as by increasing apodeme surface area. Muscle stress may be viewed as a fine control to torque generation, while apodeme surface area provides coarse control. The latter sets mechanical and spatial limits to force production while the former is under the animal's control to allow fine-tuning and graded control of force and torque. The moment arm may also contribute significantly to the magnitude of torque generation and in this case it appears to largely control the distribution of torque through the step cycle. Lieber and Boakes (1988a) suggest that muscle force is more significant than moment arm in torque generation by the frog Rana pipiens, though they also maintain that both interact in the torque production (Lieber and Boakes, 1988b). Lieber (1997) further suggests a matching of muscle fibre length and moment arm to maximize torque in the mouse. In the case of the crustaceans reported here, it appears that the moment arm is largely responsible for the torque, as the muscle force is unlikely to change appreciably over the course of the stepping cycle (assuming, of course that the muscle stress is not significantly time-varying).

This study, in concert with the two preceding reports (Mitchell and DeMont, 2003a,b), has documented muscle architecture, morphometrics, kinematics and allometry of the mero-carpopodite joint of the walking legs of the American lobster and snow crab. By synthesizing all of this information, it has been possible to accurately model force generation and torque development in these walking crustaceans and to begin to ascribe relative importance to the various model variables. Future work is required largely in identifying/documenting whole muscle characteristics of these animals during walking to refine estimates of muscle stress and the time course of the development of the muscle contraction. These reports have provided a nearcomprehensive assessment of a single joint system for two crustaceans locomoting in different manners and the findings contribute to the existing bodies of information from a number of subject areas - muscle architecture, scaling, kinematics, and mechanics.

\section{Acknowledgements}

We would like to acknowledge the assistance and support of Mikio Moriyasu and Marc Lanteigne of the Department of Fisheries and Oceans, Moncton, New Brunswick, and Jim Williams of St. Francis Xavier University, Antigonish, Nova Scotia for early review of the manuscript. Also we thank two anonymous reviewers for their constructive 
criticism. Funding for this work was provided by a Natural Sciences and Engineering Research Council of Canada grant to E. DeMont.

\section{References}

Alexander, R.McN. (1969). Mechanics of the feeding action of a cyprinid fish. J. Zoology. Lond., 159, 1-15. Alexander, R.McN. (1983). Animal Mechanics, 2nd Edn. p. 301. Blackwell Scientific Publications, London. Alexander, R.McN. (1985). The maximum forces exerted by animals. J. Exp. Biol., 115, 231-238

Atwood, H.L. (1972). Crustacean muscle. In: (Ed.) Bourne, G. The Structure and Function of Muscle, Vol. 1, pp. 421-489. Academic Press, New York.

Atwood, H.L. (1973). An attempt to account for the diversity of crustacean muscles. Am. Zoologist, 13, $357-378$.

Ayers, J. and Clarac, F. (1978). Neuromuscular strategies underlying different behavioural acts in a multifunctional crustacean leg joint. J. Comp. Physiol., 128, 81-94.

Ayers, J.L. and Davis, W.J. (1977). Neuronal control of locomotion in the lobster, Homarus americanus. I. Motor programs for forward and backward walking. J. Comp. Physiol., 115, 1-27.

Bennet-Clark, H.C. (1975). The energetics of the jump of the locust Schistocerca gregaria. J. Exp. Biol., 63, 53-83.

Bevington, P.R. (1969). Data Reduction and Error Analysis for the Physical Sciences, p. 336. McGraw-Hill Book Company, New York.

Blickhan, R. and Full, R.J. (1987). Locomotion energetics of the ghost crab II. Mechanics of the center of mass during walking and running. J. Exp. Biol., 130, 155-174.

Blickhan, R., Full, R.J. and Ting, L. (1993). Exoskeletal strain: evidence for a trot gallop transition in rapidly running ghost crabs. J. Exp. Biol., 179:301-329.

Blundon, J.A. (1988). Morphology and muscle stress in chelae of temperate and tropical stone crabs Menippe mercenaria. J. Zool., London, 215, 663-673.

Chasserat, C. and Clarac, F. (1983). Quantitative analysis of walking in a decapod crustacean, the rock lobster Jasus lalandii. J. Exp. Biol., 107, 219-243.

Clarac, F. (1981). Postural reflexes coordinating walking legs in a rock lobster. J. Exp. Biol., 90, 333-337.

Clarac, F. (1984). Spatial and temporal co-ordination during walking in crustacea. Trends in Neuroscience. 7, 293-298.

Clarac, F. and Chasserat, C. (1983). Quantitative analysis of walking in decapod crustacean, the rock lobster Jasus lalandii. 1. Comparative study of free and driven walking. J. Exp. Biol., 107, 189-217.

Clarac, F. and Cruse, H. (1982). Comparison of forces developed by the leg of the rock lobster when walking free or on a treadmill. Biol. Cybernetics, 43, 109-114.

Daniel, T.L. and Meyhöfer, E. (1989). Size limits in escape locomotion of Carridean shrimp. J. Exp. Biol., 143, 245-265.

Elner, R.W. and Campbell, A. (1981). Force, function and mechanical advantage in the chelae of the American lobster Homarus americanus (Decapoda: Crustacea). J. Zool., London, 193, 269-286.

Evoy, W.H. and Ayers, J. (1982). Locomotion and control of limb movements. In: E.D. Bliss (Ed.), The Biology of Crustacea, Vol. 4, pp. 61-105. Academic Press, New York.

Gans, C. (1982). Fiber architecture and muscle function. Exercise and Sport Science Review, 10, 160-207.

Gans, C. and deVree, F. (1987). Functional bases of fibre length and angulation in muscle. J. Morphol., 192, 63-85.

Goslow, G.E. Jr. and Van de Graaff, K.M. (1982). Hindlimb joint angle changes and action of the primary ankle extensor muscles during posture and locomotion of the striped skunk (Mephitis mephitis). J. Zool., London, 197, 405-419.

Govind, C.K. (1995). Muscles and their innervation. In: J.R. Factor (Ed.), Biology of the Llobster Homarus amercanus. pp. 291-312. Academic Press, New York.

Govind, C.K. and Blundon, J.A. (1985). Form and function of the asymmetric chelae in blue crabs with normal and reversed handedness. Biol. Bull., 168, 321-331.

Hui, C.A. (1992). Walking of the shore crab Pachygrapsus crassipes in its two natural environments. J. Exp. Biol., 165, 213-227.

Jahromi, S.S. and Atwood, H.L. (1969). Correlation of structure, speed of contraction, and total tension in fast and slow abdominal muscle fibres of the lobster (Homarus americanus). J. Exp. Zool., 171, 25-38.

Jahromi, S.S. and Atwood, H.L. (1971). Structural and contractile properties of lobster leg muscle fibers. J. Exp. Zool., 176, 475-486.

Jamon, M. and Clarac, F. (1997). Variability of leg kinematics in free-walking crayfish, Procambrus clarkii, and related inter-joint coordination. J. Exp. Biol., 200, 1201-1213. 
Josephson, R.K. and Stokes, D.R. (1987). The contractile properties of a crab respiratory muscle. J. Exp. Biol., 131, 265-287.

Josephson, R.K. and Stokes, D.R. (1989). Strain, muscle length and work output in a crab muscle. J. Exp. Biol., 145, 45-61.

Josephson, R.K. and Stokes, D.R. (1999). The force-velocity properties of a crustacean muscle during lengthening. J. Exp. Biol., 202, 593-607.

Klärner, D. and Barnes, W.J.P. (1986). The cuticular stress detector (CSD2) of the crayfish. II activity during walking and influence on leg coordination. J. Exp. Biol., 122, 161-175.

Ledoux, W.R., Hirsch, B.E., Church, T. and Caunin, M. (2001). Pennation angles of the intrinsic muscles of the foot. J. Biomechanics. 34, 399-403.

Lieber, R.L. (1997). Muscle fiber length and moment arm coordination during dorsi- and plantarflexion in the mouse hindlimb. Acta Anat., 159, 84-89.

Lieber, R.L and Boakes, J.L. (1988a). Muscle force and moment arm contributions to torque production in frog hindlimb. Am. J. Physiol., 254, C769-C772.

Lieber, R.L and Boakes, J.L. (1988b). Sarcomere length and joint kinematics during torque production in frog hindlimb. Am. J. Physiol., 254, C759-C768.

Macmillan, D.L. (1975). A physiological analysis of walking in the American lobster (Homarus americanus). Phil. Trans. Roy. Soc. B., 270, 1-59.

Macmillan, D.L. and Dando, M.R. (1972). Tension receptors on the apodemes of muscles in the walking legs of the crab Cancer magister. Mar. Behav. Physiol., 1, 185-208.

Martinez, M.M., Full, R.J. and Koehl, M.A.R. (1998). Underwater punting by an intertidal crab: a novel gait revealed by the kinematics of pedestrian locomotion in air versus water. J. Exp. Biol., 201, 2609-2623.

Mitchell, S. and DeMont, M.E. (2003a). Analysis of the mero-carpopodite joint of the American lobster and snow crab. I. Apodeme surface area and muscle fibre pinnation angle. J. Mar. Biol. Ass. U.K., 83, 1239-1248.

Mitchell, S. and DeMont, M.E. (2003b). Analysis of the mero-carpopodite joint of the American lobster and snow crab. II. Kinematics, morphometrics, and moment arms. J. Mar. Biol. Ass. U. K., 83, 1249-1259.

Nauen, J.C. and Shadwick, R.E. (1999). The scaling of acceleratory locomotion: body size and tail-flip performance of the California spiny lobster Panulirus interruptus. J. Exp. Biol., 202, 3181-3193.

Pond, C.M. (1975). The role of the 'walking legs' in aquatic and terrestrial locomotion of the crayfish Austropotomobius pallipes (Lereboullet). J. Exp. Biol., 62, 447-454.

Prosser, C.L. and Brown, F.A. (1962). Comparative Animal Physiology. p. 688, 2nd Edn. W. B. Saunders Company, Philadelphia.

Richmond, F.J.R. (1998). Elements of style in neuromuscular architecture. Am. Zoologist, 38, 729-742.

Sherman, R.G. and Atwood, H.L. (1971). Structure and neuromuscular physiology of a newly discovered muscle in the walking legs of the lobster Homarus americanus. J. Exp Zool., 176, 461-474.

Sleinis, S. and Silvey, G.E. (1980). Locomotion in a forward walking crab. J. Comp. Physiol., 136, $301-312$.

Taylor, G.M. (2000). Maximum force production: why are crabs so strong? Proc. Roy. Soc. Lond. B., 267, $1475-1480$.

Warner, G.F. and Jones, A.R. (1976). Leverage and muscle type in crab chelae (Crustacea:Brachyura). J. Zool., 180, 57-68.

Warner, G.F., Chapman, D., Hawkeye, N. and Waring, D.G. (1982). Structure and function of the chelae and chela closer muscles of the shore crab Carcinus maenas (Crustacea: Brachyura). J. Zool, London, 96, 431-438.

Webb, P.W. (1979). Mechanics of escape responses in crayfish (Orconectes virilis). J. Exp. Biol., 79, $245-263$.

Zachar, J. and Zacharova, D. (1966). Potassium contractures in single muscle fibres of the crayfish. J. Physiol., 186, 596-618. 